\title{
Atorvastatin Inhibits Myocardial Apoptosis in a Swine Model of Coronary Microembolization by Regulating PTEN/ PI3K/Akt Signaling Pathway
}

\author{
Jiangyou Wang ${ }^{a}$ Han Chen ${ }^{b}$ You Zhou ${ }^{a}$ Qiang Su ${ }^{a}$ Tao Liu ${ }^{a}$ Xian-tao Wang \\ Lang Lia \\ aDepartment of Cardiology, the First Affiliated Hospital of Guangxi Medical University, Guangxi \\ Cardiovascular Institute, Nanning, 'bepartment of Cardiac Surgery, Wuhan Asia Heart Hospital, Wuhan, \\ China
}

\author{
Key Words \\ PTEN • PI3K • Akt • Atorvastatin • Coronary microembolization • Apoptosis
}

\begin{abstract}
Background/Aims: Phosphatase and tensin homolog deleted on chromosome ten (PTEN) has been recognized as a promoter of apoptosis in various tissues, and revealed to be upregulated in circumstances of coronary microembolization (CME). However, whether this functional protein could be modified by pretreatment of atorvastatin in models of CME has not been disclosed yet. Methods: Swine CME was induced by intra-coronary injection of inertia plastic microspheres (diameter $42 \mu \mathrm{m}$ ) into left anterior descending coronary, with or without pretreatment of atorvastatin or PTEN siRNA. Echocardiologic measurements, pathologic examination, TUNEL staining and western blotting were applied to assess their functional, morphological and molecular effects in CME. Results: PTEN were aberrantly upregulated in cardiomyocytes following $C M E$, with both the $\mathrm{mRNA}$ and protein levels increased after CME modeling. Pretreatment with atorvastatin could attenuate the induction of PTEN. Furthermore, down-regulation of PTEN in vivo via siRNA was associated with an improved cardiac function, attenuated myocardial apoptosis, and concomitantly inhibited expressions of key proapoptotic proteins such as Bax, cleaved-caspase-3. Interestingly, atorvastatin could markedly attenuate PTEN expression and therefore partially reverse cardiac dysfunction and attenuate the apoptosis of the myocardium following CME. Conclusion: Modulation of PTEN was probably as a potential mechanism involved in the beneficial effects of pretreatment of atorvastatin to cardiac function and apoptosis in large animal models of CME.
\end{abstract}




\section{Cellular Physiology Cell Physiol Biochem 2016;38:207-219

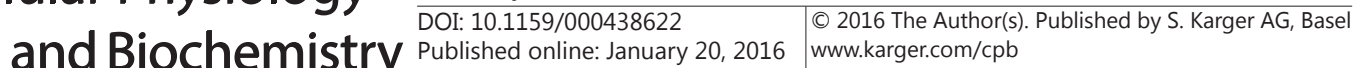 \\ Wang et al.: Atorvastatin is Involved in CME-Induced Myocardial Apoptosis}

\section{Introduction}

Coronary microembolization (CME), which could be induced by either a spontaneous rupture of a vulnerable coronary atherosclerotic plaque or therapeutic percutaneous coronary interventions (PCI), has been found be associated with adverse outcomes such as cardiac contractile dysfunction, and reduced coronary reserve [1]. There have been major advances in identifying the factors of the myocardial inflammatory response and apoptosis that are involved in CME-induced myocardial injury [2-7]. However, the overall complexity of myocardial injury suggests that additional regulatory mechanisms remain to be elucidated. Therefore, it is reasonable to propose that interventions which attenuate myocardial apoptosis could be protective for the heart from CME related cardiac dysfunction. Statins, the most widely prescribed cholesterol-lowering agents, have been suggested to be antiapoptotic in myocardium, possibly via multiple mechanisms [8]. Also, a previous study by Herrmann et al. [9] showed that a beneficial effect of statin against peri-procedural coronary microembolization. And, results of our recent studies revealed that atorvastatin could lower the extent of myocardial injury after CME and improve the cardiac function in mice primarily related to the regulation of cardiomyocytes apoptosis [10], although the potential molecular mechanisms remained unclear.

Phosphatase and tensin homolog deleted on chromosome ten (PTEN), a negative regulator of phosphatidylinositol-3 kinase (PI3K)-Akt pathways, has been recently found to be up-regulated in ischemic myocardium and involved in a wide variety of myocardial apoptotic responses [11]. The PTEN/Akt pathway had been involved in the pathogenesis of many cardiovascular diseases such as cardiac hypertrophy, myocardial contractile dysfunction, coronary angiogenesis, heart failure and ischemia-reperfusion injury [12]. However, whether the protective effects of atorvastatin to CME related myocardial apoptosis were related to the regulation of the PTEN/Akt pathway remains to be determined.

\section{Materials and Methods}

\section{Animal preparation}

Healthy swine (25 - $30 \mathrm{~kg}$ ) were purchased from the Animal Center of the Agriculture College of Guangxi University (Nanning, People's Republic of China), and throughout all of the experimental stages, the animals were maintained under controlled temperature, humidity, and light conditions, with pig feed and water provided ad libitum. This investigation conforms to the Guide for the Care and Use of Laboratory Animals published by the US National Institute of Health (NIH publication No. 85-23, revised 1996). The Clinical and Animal Research Ethics Committees of Guangxi Medical University approved all of the procedures.

\section{Generation of the CME model}

The CME model was induced by the manual unremitting injection of microspheres into the left anterior descending (LAD) artery, as previously described by Dorge et al. [4] Breuckmann et al. [13] and Carlsson et al. [14].The swine were initially sedated via an IM injection of a combination of ketamine and atropine (10 - $15 \mathrm{mg} / \mathrm{kg}$ and $2 \mathrm{mg}$, respectively). After endotracheal intubation, anesthesia was maintained via an intravenous drip of diazepam into the ear vein. The right femoral artery was separated, and a 6F (Cordis, USA) vascular sheath was placed. Prior to the coronary cannulation, the animals were anticoagulated via an intravenous injection of $200 \mathrm{U} / \mathrm{kg}$ heparin followed by $100 \mathrm{U} / \mathrm{kg}$ per h to maintain heparinization. A $6 \mathrm{~F}$ JL 4.0 guiding catheter was used for the coronary angiography. After the coronary angiography, a $1.8 \mathrm{~F}$ infusion catheter (Cordis, Inc., USA) was placed into the left anterior descending (LAD) artery with the tip located between the second and third diagonal branches. Microspheres with a diameter of $42 \mu \mathrm{m}$ (Dynospheres; Dyno Particles; Lillestrøm, Norway) at a mean dosage of 100,000 were selectively infused into the LAD within $40 \mathrm{~min}$ followed by a flush with $10 \mathrm{ml}$ of saline. The sham-operated swine (sham group) were subjected to the same procedures, except that the injection was saline rather than microspheres. The systemic blood pressure and heart rate were continuously monitored during the procedure. 


\section{Cellular Physiology Cell Physiol Biochem 2016;38:207-219

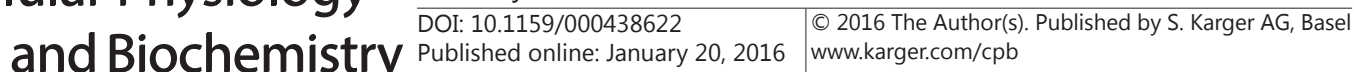 \\ Wang et al.: Atorvastatin is Involved in CME-Induced Myocardial Apoptosis}

In vivo PTEN siRNA administration

The swine were randomly distributed into four groups as follows: the sham surgery group (control, $\mathrm{n}=$ 8), CME group (CME, $n=8$ ), PTEN siRNA-treated before CME group (PTEN siRNA, $n=8$ ), and control siRNAtreated group (control siRNA, $\mathrm{n}=8$ ). In vivo transfer was performed according to the method described previously [13]. After coronary angiography, a 1.8F infusion catheter (Cordis, Inc. USA) was placed in the LAD artery with the tip located between the second and third diagonal branches. A total of $100 \mu \mathrm{g}$ of PTEN siRNA (Genepharma, PTEN-sus-368) or control siRNA (Genepharma) was diluted in the same volume of the in vivo transfection reagent (EntransterTM-in vivo; Engreen, Beijing, China). After mixing the solution gently by pipetting up and down, the siRNA solutions were selectively infused into the LAD. Then, $72 \mathrm{~h}$ after the PTEN siRNA or control siRNA administration, CME surgery was performed. In our previous study, we found that the protein level of PTEN was obviously up-regulated at $12 \mathrm{~h}$ post-CME [6], so all of the swine were sacrificed at $12 \mathrm{~h}$ after the CME or sham surgery in this study.

Design, synthesis and transfection of PTEN SIRNA

The PTEN mRNA sequence was found in GenBank (Gene ID: 100156264) and synthesized by GenePharma company (Shanghai, China). The sense strand and the antisense strand of the PTEN siRNA were 5'-GGC GUA UAC AGG AAC AAU ATT-3' and 5'- UAU UGU UCC UGU AUA CGC CTT-3', respectively. The control siRNA was purchased from the same company. A total mass of $100 \mu \mathrm{g}$ PTEN siRNA or control siRNA was diluted with $100 \mu \mathrm{l}$ transfection reagent (EntransterTM - in vivo; Engreen, Beijing, China). Mix thoroughly by pipetting the solution up and down. Then the mixture was injected intracoronarily via the $1.8 \mathrm{~F}$ infusion catheter, followed by $2 \mathrm{ml}$ normal saline injection.

Serum c-troponin I measurement

EDTA-anticoagulated blood samples $(1.0 \mathrm{ml})$ were collected from the femoral artery in each group 12 $\mathrm{h}$ following sham operation or CME and centrifuged at $4000 \mathrm{rpm}$ for $15 \mathrm{~min}$. Serum samples were stored at $-80^{\circ} \mathrm{C}$ for detection. Serum c-troponin I was detected by electro-chemistry method according to the manufacturer's instruction (Roche, Inc, Switzerland).

\section{Echocardiography}

Transthoracic echocardiographic studies were performed by one experienced investigator who was blinded to the study protocol using a GE VIVID 7 system with a 1.5-4.3 MHZ transducer. Left ventricle ejection fraction (LVEF), left ventricle end-diastolic diameter (LVEDd), fractional shortening (FS) and cardiac output (CO) were obtained.

Tissue sampling

After blood sample collection, the hearts were arrested in diastole via injection of $10 \mathrm{ml}$ of $10 \%$ potassium chloride into the ear vein. Hearts were isolated and cleaned with normal saline immediately. Myocardial tissues were obtained from the anterior wall of left ventricle dominated by the middle of the LAD artery. Part of myocardial tissue was immediately frozen in liquid nitrogen and stored at $-80^{\circ} \mathrm{C}$ for Western blot analysis. The other was fixed with $4 \%$ paraformaldehyde for $12 \mathrm{~h}$, embedded in paraffin and serially sectioned into slices of $4 \mu \mathrm{m}$ thickness for Hematoxylin basic fuchsin picric acid (HBFP) staining and Immunohistochemical staining and Terminal deoxymu-cleotidyl Transferase-Mediated dUTP Nick EndLabeling (TUNEL) assay.

Measuring the myocardial microinfarction area

HBFP (haematoxylin fuchsin basic picric acid staining) staining is an important method for the early diagnosis of myocardial ischemia. HBFP stains ischemic cardiac muscle, normal myocardial cytoplasm, and nuclei red, yellow, and blue, respectively. A DMR-Q550 pathological image pattern analysis instrument (Leica, Germany) was used to analyse the HBFP-stained slices. Briefly, five microscopic visual fields (original magnification, $\times 100$ ) were randomly sampled from each slice for analysis using QWin analysis software (Leica, Germany), and the planar area method was used to measure the infarction zone, which was expressed as the average percentage of the area of infarction out of the total analysed slice area.

\section{KARGER}




\section{Cellular Physiology Cell Physiol Biochem 2016;38:207-219 and Biochemistry \begin{tabular}{l|l} 
DOI: 10.1159/000438622 & (c) 2016 The Author(s). Published by S. Karger AG, Basel \\
www.karger.com/cpb
\end{tabular} \\ Wang et al.: Atorvastatin is Involved in CME-Induced Myocardial Apoptosis}

TUNEL assay

Apoptotic cardiomyocytes were detected using the terminal deoxymu-cleotidyl transferase-mediated dUTP nick end-labeling (TUNEL) assay kit (Roche, USA). TUNEL-positive signal was located in nuclei and apoptotic nuclei were stained yellow-brown while the normal cell nuclei light blue. Meanwhile, morphological features of apoptosis (small, condensed nuclei, cell shrinkage and nuclear fragmentation) were taken into consideration. In each section, 10 random high-powered fields (magnification, $\times 400$ ) were observed to count TUNEL-positive cardiomyocyte nuclei and apoptotic index (\%) was calculated as the percentage ratio of TUNEL-positive cell nuclei to the total nuclei.

\section{Real-time quantitative PCR analysis}

Total RNA from a fresh tissue block was extracted using a Trizol Reagent Kit (Gibco, USA), and 4 $\mu \mathrm{g}$ of RNA was reverse-transcribed using a Reverse Transcriptase Kit (Promega, USA) according to the manufacturer's instructions.

Real-time PCR was performed using an ABI PRISM 7000 system (Applied Biosystems, CA, USA) using the non-sequence-specific SYBR Green I dye (TaKaRa, Japan). The reactions included $2 \mu \mathrm{l}$ of cDNA, primers ( $0.5 \mu \mathrm{l}$ each), and $12.5 \mu \mathrm{l}$ of SYBR Green Master Mix (Applied Biosystems, CA, USA), which binds to any doublestranded DNA, as well as water added to a final volume of $25 \mu$ l. PCR was performed under the following cycling conditions: initial denaturation step at $95^{\circ} \mathrm{C}$ for $10 \mathrm{~min}$, followed by 45 cycles of amplification $\left(95^{\circ} \mathrm{C}\right.$ for $15 \mathrm{~s}$ and $60^{\circ} \mathrm{C}$ for $1 \mathrm{~min}$ ). The primers were 5-GAG CCA TTT CCA TCC TGCAG-3 and 5-GCT GTC ATG TCT GGG AGTCT-3 for swine PTEN and 5-CAC CTT CTA CAA CGA GCTGC-3 and 5-TCA TCT TCT CAC GGT TGGCT-3 for actin (all primers were designed by TaKaRa, Japan). The transcript expression levels were quantified via the Ct value method, and the Ct values were normalised to those of actin as an internal control in the same sample. The specificity of the amplified PCR products was confirmed based on the melting curves.

\section{Western blot analysis}

Briefly, myocardial tissues were homogenized, and aliquots of each fraction were used to determine the protein concentration of each sample using a detergent compatible assay. Protein samples (100 $\mu \mathrm{g})$ were loaded onto polyacrylamide gel, electrophoresed, and transferred to a nitrocellulose membrane. The nitrocellulose membranes were then blocked followed by incubation with the primary antibodies overnight at $4^{\circ} \mathrm{C}$. The following primary antibodies were purchased from Abcam: (1) rabbit anti-PTEN, (2) rabbit anti-PI3K, p-PI3K, (3) rabbit anti-Akt, p-Akt, (4) rabbit anti-Bax and Bcl-2, and (5) rabbit anti-cleaved caspase-3. Immunoblots were processed with secondary antibodies (Abcam Biotechnology) for $1 \mathrm{~h}$ at room temperature. Immunoblots were probed and then exposed to X-ray film. The X-ray films were scanned, and the optical density was determined by Bio-Rad image analysis (Bio-Rad, Hercules, CA, USA).

\section{Statistical analysis}

Statistical analyses were performed using SPSS 13.0 statistical software (IBM Corporation, USA). The quantitative data are presented as the mean (SD). Unpaired Student's t-test and ANOVA were used for comparisons between two groups and among multiple groups, respectively, followed by the StudentNewman-Keuls test for the post hoc analysis. The $P<0.05$ level indicated statistical significance.

\section{Results}

\section{Animal groups}

No significant differences in the body weight or heart rate were observed among the four groups (data not shown).

Atorvastatin and PTEN siRNA pretreatments improved cardiac function following CME

Results of echocardiographic examination (Table 1) showed that $12 \mathrm{~h}$ after CME modeling, the CME group exhibited significantly decreased cardiac systolic function as compared with the sham group, as indicated by the significant reduced LVEF, FS and CO, as well as increased LVEDd in CME group $(P<0.05)$. Atorvastatin pretreatment improved all indices of cardiac function in CME-treated animals, as reflected by the increased LVEF, FS and 
Table 1. Parameters of cardiac function in minipigs of each group $12 \mathrm{~h}$ after CME modeling: results of the Echocardiologic measurements. CME, coronary microembolization; LVEF, left ventricular ejection fraction; FS, fractional shortening; LVEDd, left ventricular end-diastolic diameter; CO, cardiac output; ATV, atorvastatin; PTEN, phosphatase and tensin homolog deleted on chromosome ten. Data are presented as mean (SD). ${ }^{a} \mathrm{P}<0.05$ compared with sham; ${ }^{\text {b }}<0.05$ compared with CME or control siRNA $(\mathrm{N}=8)$

\begin{tabular}{lllll}
\hline Groups & LVEF $(\%)$ & FS $(\%)$ & LVEDd $(\mathrm{mm})$ & CO $(\mathrm{L} / \mathrm{min})$ \\
\hline sham & $69.74(2.85)$ & $42.18(2.28)$ & $31.70(1.63)$ & $4.16(0.28)$ \\
CME & $49.75(4.25)^{\mathrm{a}}$ & $24.57(3.24)^{\mathrm{a}}$ & $38.35(1.43)^{\mathrm{a}}$ & $2.49(0.27)^{\mathrm{a}}$ \\
ATV & $59.26(2.59)^{\mathrm{b}}$ & $31.20(2.52)^{\mathrm{b}}$ & $34.21(1.54)$ & $3.42(0.24)$ \\
PTEN siRNA & $58.37(4.56)^{\mathrm{ab}}$ & $30.45(3.62)^{\mathrm{ab}}$ & $33.25(1.28)$ & $3.47(0.33)^{\mathrm{b}}$ \\
Control siRNA $^{4}$ & $48.28(3.39)^{\mathrm{a}}$ & $25.62(3.23)^{\mathrm{a}}$ & $39.16(1.25)^{\mathrm{a}}$ & $2.53(0.32)^{\mathrm{a}}$ \\
\hline
\end{tabular}

Fig. 1. Parameters of cTnI in minipigs of each group $12 \mathrm{~h}$ after CME modeling: CME, coronary microembolization; ATV, atorvastatin; PTEN, phosphatase and tensin homolog deleted on chromosome ten. ${ }^{a} P<0.05$ compared with sham; ${ }^{b} P<0.05$ compared with CME or control siRNA $(\mathrm{N}=8)$.

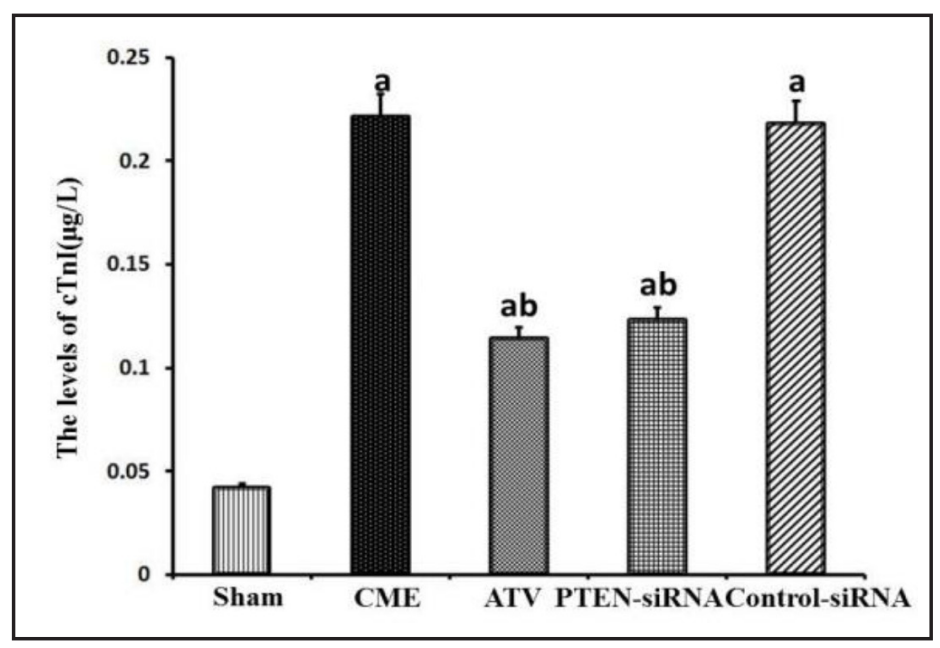

CO, as well as decreased LVEDd of the ATV group as compared with the CME group $(P<0.05)$. Similarly, PTEN siRNA pretreatment was also associated with improved cardiac function in CME swine.

Atorvastatin and PTEN siRNA pretreatments attenuated mocardial injury following CME Myocardial injury following CME could be assessed by levels of cTnI in the blood obtained from coronary sinus (Fig. 1). At $12 \mathrm{~h}$ after CME modeling, serum level of cTnI in swine from CME group or control siRNA were higher than controls [0.221 (0.018) $\mu \mathrm{g} / \mathrm{L}$ vs $0.042(0.008) \mu \mathrm{g} / \mathrm{L}, p<0.05]$. Moreover, atorvastatin pretreatment attenuated myocardial injury following CME, as reflected by the reduced cTnI levels in ATV group compared with CME group [0.114 (0.012) $\mu \mathrm{g} / \mathrm{L}$ vs $0.221(0.018) \mu \mathrm{g} / \mathrm{L}, p<0.05]$. In addition, PTEN siRNA pretreatment was also associated with reduced cTnI levels $[0.123(0.015) \mu \mathrm{g} / \mathrm{L}]$, which was similar to the effects of atorvastatin pretreatment. No significant differences in the levels of cTnI was observed between the CME and Control siRNA groups $[0.221(0.018) \mu \mathrm{g} / \mathrm{L}$ vs 0.218 (0.019) $\mu \mathrm{g} / \mathrm{L}, p>0.05]$.

\section{CME Histopathology}

As revealed by Mayer's haematoxylin and eosin (H\&E) and haematoxylin-basic fuchsinpicric acid (HBFP) staining (Fig. 2). The sham control animals exhibited subendocardial ischemia without infarction foci, whereas the CME animals exhibited multiple microinfarction foci. However, the administration of ATV reduced the microinfarct volume and inflammatory cell infiltration. HBFP staining revealed myocardial karyolysis or hypochromatosis based on the red cytoplasmic staining of the microinfarction foci. In addition, peripheral cardiac 


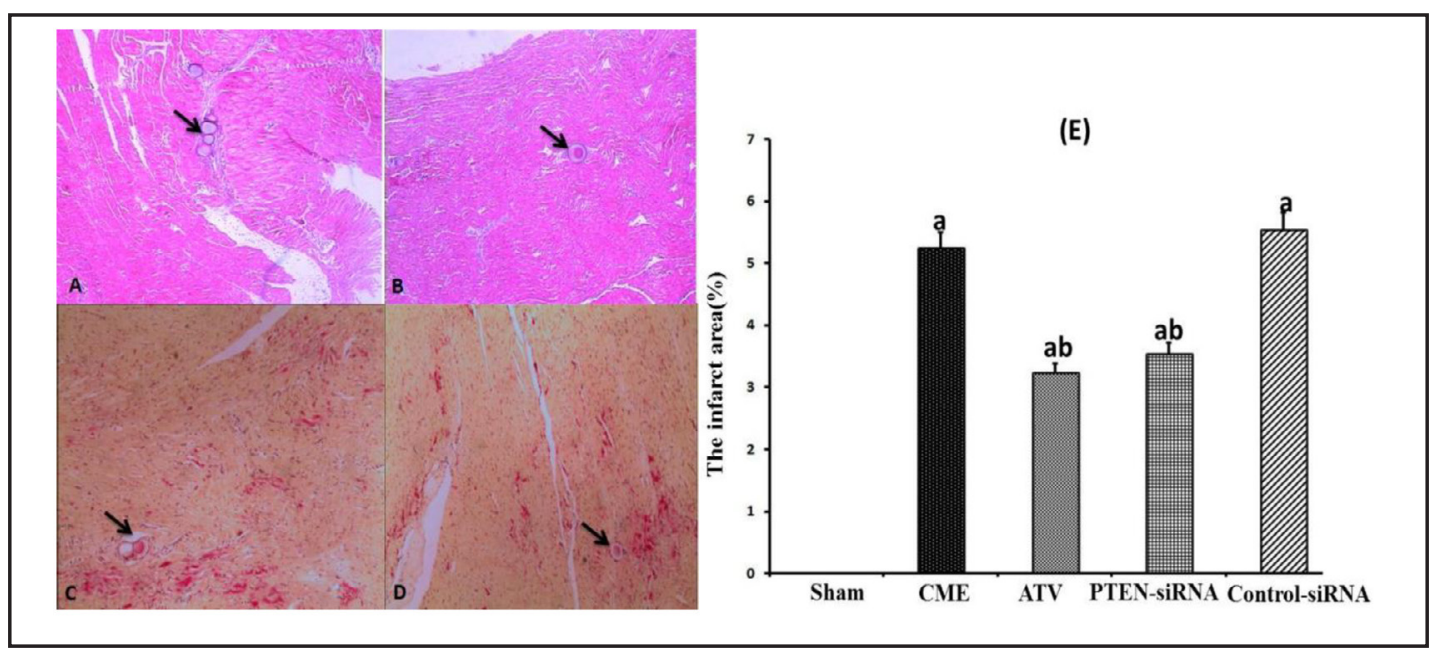

Fig. 2. (A, B) H\&E staining of tissue samples from the CME group revealed myocardial microinfarcts displaying a high level of inflammatory cell infiltration (A, 12 h post-CME). However, the administration of ATV reduced the microinfarct volume and inflammatory cell infiltration (B). The arrow indicates the presence of a $42 \mu \mathrm{m}$ microsphere following CME (H\&E staining; $\times 100$ ). (C, D) HBFP staining of tissue samples revealed microinfarcts in the tissue sample from the CME group (C, 12_h post-CME), and a reduced microinfarct volume in the ATV-treated group (D); microinfarcts are indicated by arrows. Normal myocardial cytoplasm is stained yellow; the nuclei are stained blue; and the necrotic area is stained red (HBFP staining, $\times 100$ ). ${ }^{a} P<0.05$ compared with sham; ${ }^{b} P<0.05$ compared with CME or control siRNA, $(\mathrm{N}=8)$.

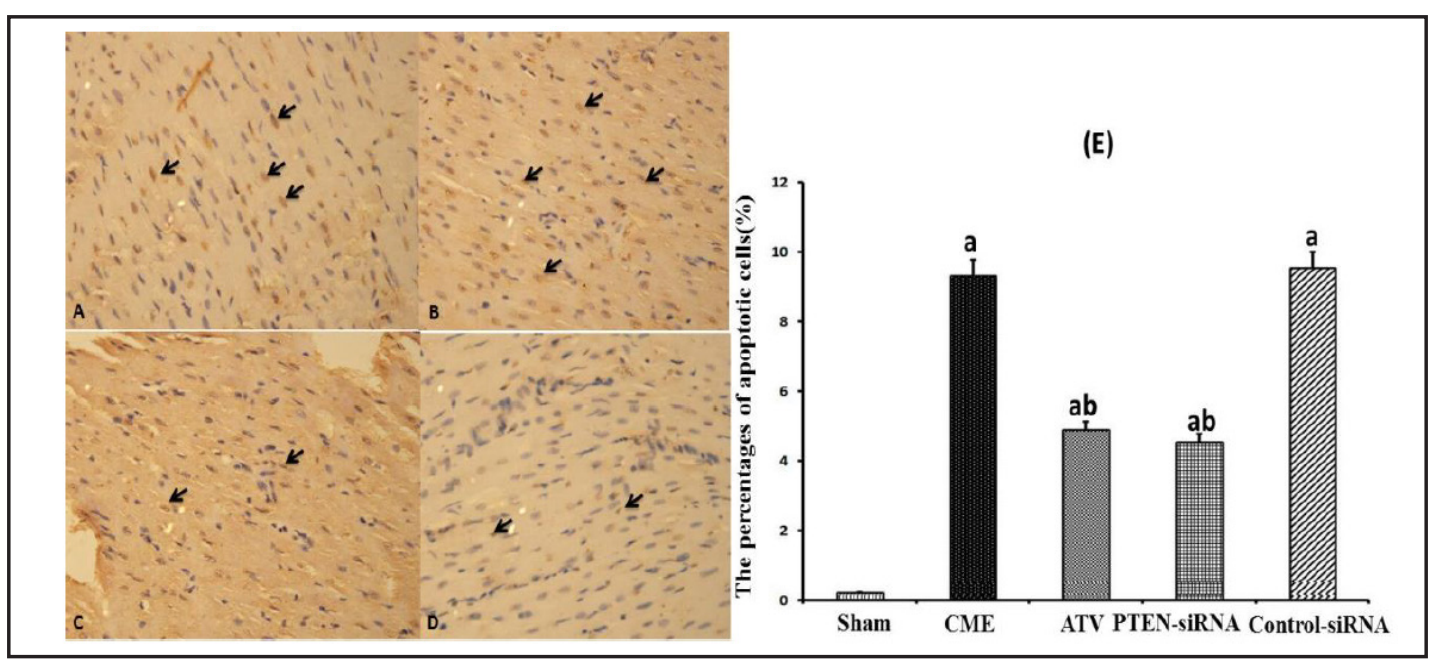

Fig. 3. Representative images and quantitative analyses of myocardial apoptosis in minipigs from each group $12 \mathrm{~h}$ post CME modeling: results of the TUNEL staining. (A) CME; (B) control siRNA; (C) PTEN siRNA; (D) ATV. Normal cell nuclei are stained in pale blue, while the apoptotic cardiomyocyte nuclei (arrows) are stained in brown $(\times 200)$. Results of quantitative analyses indicated that the apoptotic indexes of myocardium were substantially lowered in ATV and PTEN siRNA groups as compared with the CME or the control siRNA groups. ${ }^{a} p<0.05$ compared with CME or control siRNA. CME, coronary microembolization; ATV, atorvastatin; PTEN, phosphatase and tensin homolog deleted on chromosome ten.

muscle oedema and denaturation, peripheral inflammatory cell infiltration, and erythrocyte effusion were detected. For the all groups, the infarct area each group was sham $0.00(0.00)$ \%, CME 5.24 (2.65) \%, ATV 3.23(2.02) \%, PTEN siRNA 3.54 (2.35) \%, and control siRNA 5.54 (2.83) \%, respectively.

\section{KARGER}




\section{Cellular Physiology and Biochemistry Published online: January 20, $2016 \quad \begin{aligned} & \text { DOI: 10.1159/000438622 } 2016 \text { The Author(s). Published by S. Karger AG, Basel } \\ & \text { www.karger.com/cpb }\end{aligned}$ \\ Wang et al.: Atorvastatin is Involved in CME-Induced Myocardial Apoptosis}

Fig. 4. The PTEN siRNA expression in minipigs of each group $12 \mathrm{~h}$ after CME modeling: CME, coronary microembolization; ATV, atorvastatin; PTEN, phosphatase and tensin homolog deleted on chromosome ten. ${ }^{a} P<0.05$ compared with sham; ${ }^{b} P<0.05$ compared with CME or control siRNA ( $\mathrm{N}=8)$.

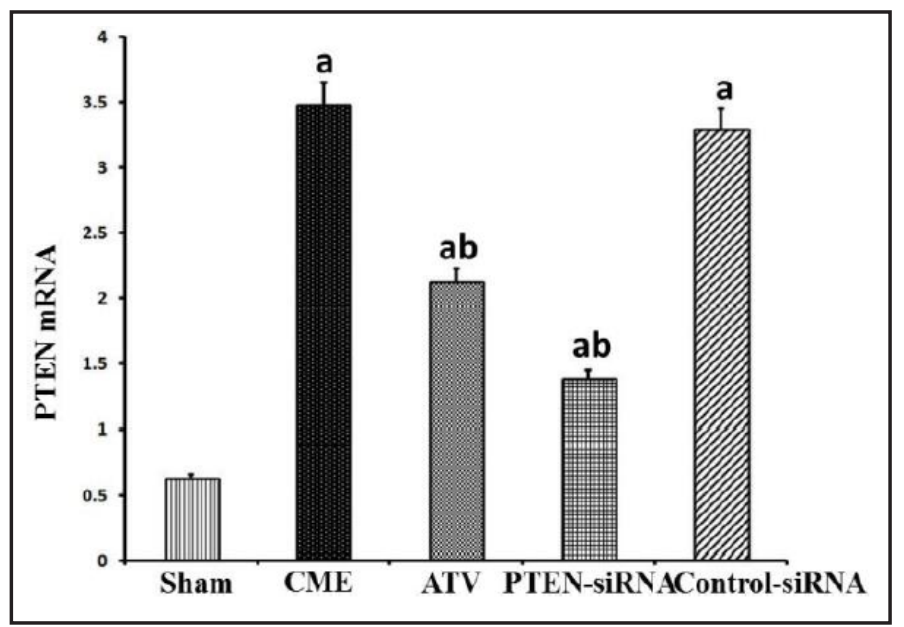

Atorvastatin and PTEN siRNA pretreatments decreased myocardial apoptosis following CME

Myocardial apoptosis was assessed using TUNEL staining. Compared with the sham group, more TUNEL-positive (brown) cardiomyocytes could be detected in swine from the CME groups $(P<0.05)$. Interestingly, atorvastatin treatment significantly decreased the relative proportion of apoptotic cells following $\operatorname{CME}(P<0.05)$. The percentages of myocardial apoptotic cells in the sham, CME, ATV, and control siRNA were 0.22 (0.16), 9.32 (1.28), 4.89 $(0.48)$ and $9.53(0.69)$ respectively (Fig. 3$)$. In addition, PTEN siRNA pretreatment was also associated with a decreased myocardial apoptosis [4.54 (0.56)], similar to the effects of atorvastatin pretreatment.

The MRNA levels of PTEN each group

The PTEN mRNA expression was significantly higher in the CME and control siRNA groups than in the sham-operated group after surgery [12 h: $3.48(0.39)$ and $3.29(0.28)$ vs. $0.63(0.12), p<0.05]$. The administration of PTEN siRNA and atorvastatin reduced the mRNA level of PTEN to 1.38(0.14) and 2.12(0.15) (Fig. 4).

PTEN siRNA pretreatment inhibited myocardial PI3K/Akt signaling in CME swine

Western blotting showed significant up-regulation of PTEN, Bax proteins, as well as a remarkable down-regulation of p-PI3K, p-Akt, Bcl-2 following CME modeling. Western blot analysis of the infarcted myocardial tissue in each group showed that the expressions of PTEN (Fig. 5A), Bax (Fig. 5D) were significantly increased in the myocardium of swine from CME groups compared with those from the sham group $(P<0.05)$. However, PTEN siRNA pretreatment was associated with reduced levels of PTEN, cleaved caspase- 3 and Bax proteins, as well as enhanced p-PI3K (Fig. 5B), p-Akt (Fig. 5C), Bcl-2 (Fig. 5D) proteins as compared with CME or control siRNA groups $(P<0.05)$.

Atorvastatin pretreatment inhibited myocardial PTEN/PI3K/Akt signaling following CME

Western blot analysis of the infarcted myocardium in each group showed that the expressions of PTEN (Fig. 6A), Bax (Fig. 6D), and cleaved caspase-3 (Fig. 6F) were significantly increased in the CME groups as compared with sham groups $(P<0.05)$. However, pretreatment of atorvastatin reduced the levels of PTEN (Fig. 6A), cleaved caspase-3 (Fig. 6F) and Bax (Fig. 6D) proteins, but enhanced the levels of p-PI3K (Fig. 6B), p-Akt (Fig. 6C), and Bcl-2 (Fig. 6E) proteins as compared with CME group $(P<0.05)$. PTEN was silenced by its siRNA then applying atorvastatin. Western blot analysis of the infarcted myocardium in each group showed that the expressions of PTEN. Atorvastatin could further inhibit the expression of PTEN protein (Fig. 6G). 


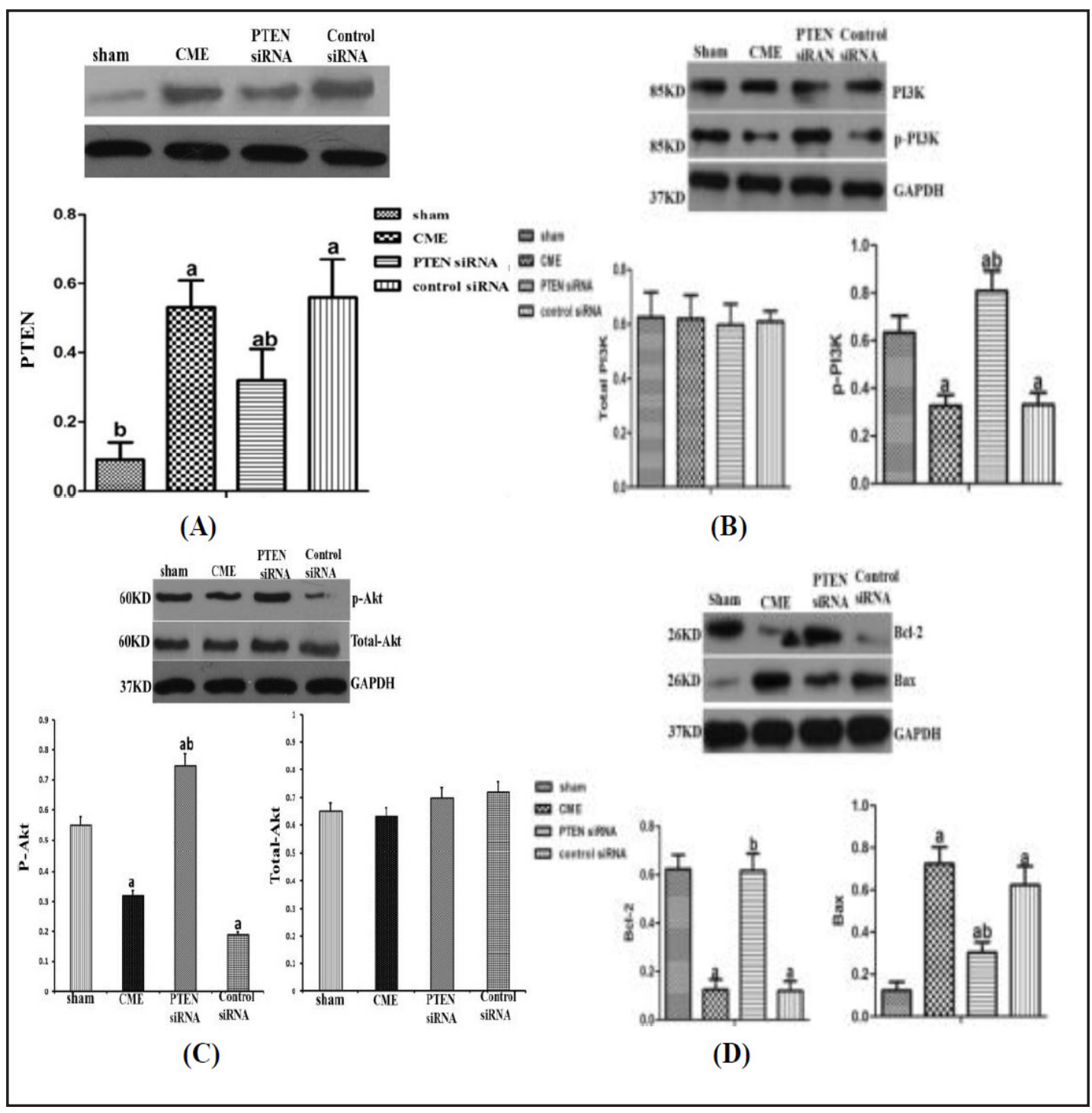

Fig. 5. Effects of CME and PTEN siRNA on the protein levels of PTEN, PI3K-Akt pathways, Bcl-2 and Bax in myocardial tissues in minipigs $12 \mathrm{~h}$ post CME modeling: results of western blotting analyses. The expression levels of PTEN (A), Bax (D), and cleaved-caspase-3 (E) were significantly increased in the CME group compared to those of the sham group ( $<<0.05)$. The expression levels of p-PI3K (B), p-Akt (C) and Bcl-2 (D) were significantly decreased in the CME group compared to those of the sham group $(\mathrm{P}<0.05)$. The expression levels of PI3K (B), Akt (C) were not difference among sham, CME and control siRNA groups. However, PTEN siRNA could reduce the levels of PTEN, Bax, cleaved-caspase-3, and enhanced the levels of p-PI3K, p-Akt and Bcl-2 as compared with the CME group or the control siRNA group. ${ }^{\mathrm{a}} \mathrm{p}<0.05$ versus sham; ${ }^{\mathrm{b}} \mathrm{P}<0.05$ versus CME or control siRNA. CME, coronary microembolization; PTEN, phosphatase and tensin homolog deleted on chromosome ten.

\section{The molecular cascade after CME}

CME induced apoptosis via up-regulation of PTEN with PI3K/Akt and cooperating with other Akt-downstream apoptotic signals, such as Bax/Bcl-2 and caspase-3. After using PTEN siRNA or atorvastatin, PI3k/Akt and other downstream genes were affected, followed by the inhibition of the apoptotic cell death process. Sham: sham group; CME: CME: CME group; PTEN siRNA: CME+ PTEN siRNA group; Control siRNA: CME+ Control siRNA group (Fig. 7). 


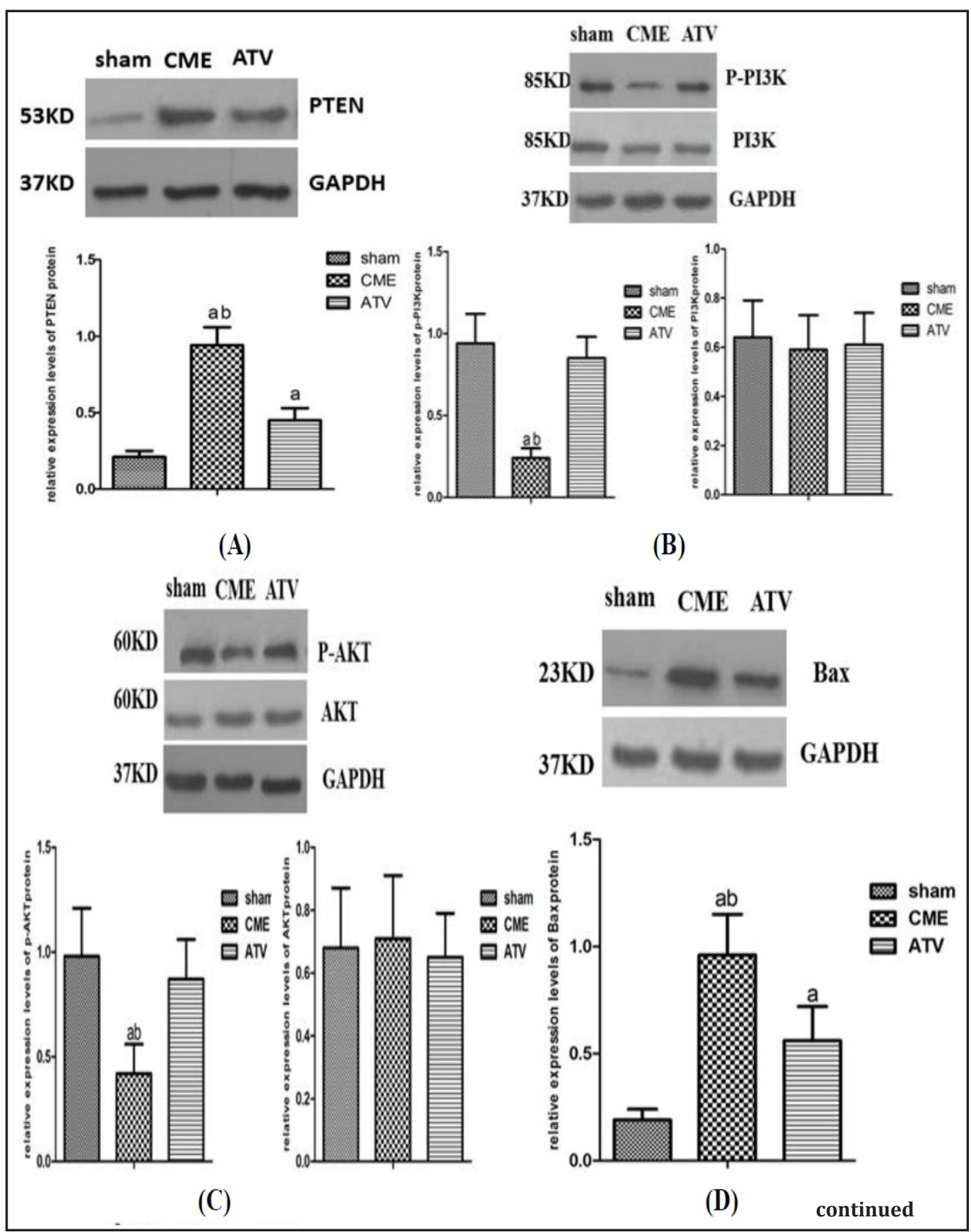

Fig. 6. Effects of CME and ATV on the protein levels of PTEN, PI3K-Akt pathways, Bcl-2 and Bax, and caspase 3 in myocardial tissues in minipigs $12 \mathrm{~h}$ post CME modeling: results of western blotting analyses. The expression levels of PTEN (A), Bax (D), and cleaved-caspase-3 (F) were significantly increased in the CME group compared to those of the sham group $(P<0.05)$. The expression levels of p-PI3K (B), p-Akt (C) and Bcl2 (E) were significantly decreased in the CME group compared to those of the sham group $(P<0.05)$. The expression levels of PI3K (B), Akt (C), and caspase-3 (F) were not difference among three groups. However, administration of atorvastatin reduced the levels of PTEN, Bax, cleaved-caspase-3 proteins, and enhanced the levels of p-PI3K, p-Akt and Bcl-2 proteins. Atorvastatin could further inhibit the expression of PTEN protein $(\mathrm{G}){ }^{\mathrm{a}} p<0.05$ versus sham; ${ }^{\mathrm{b}} P<0.05$ versus ATV. CME, coronary microembolization; PTEN, phosphatase and tensin homolog deleted on chromosome ten. ATV, atorvastatin.

\section{KARGER}




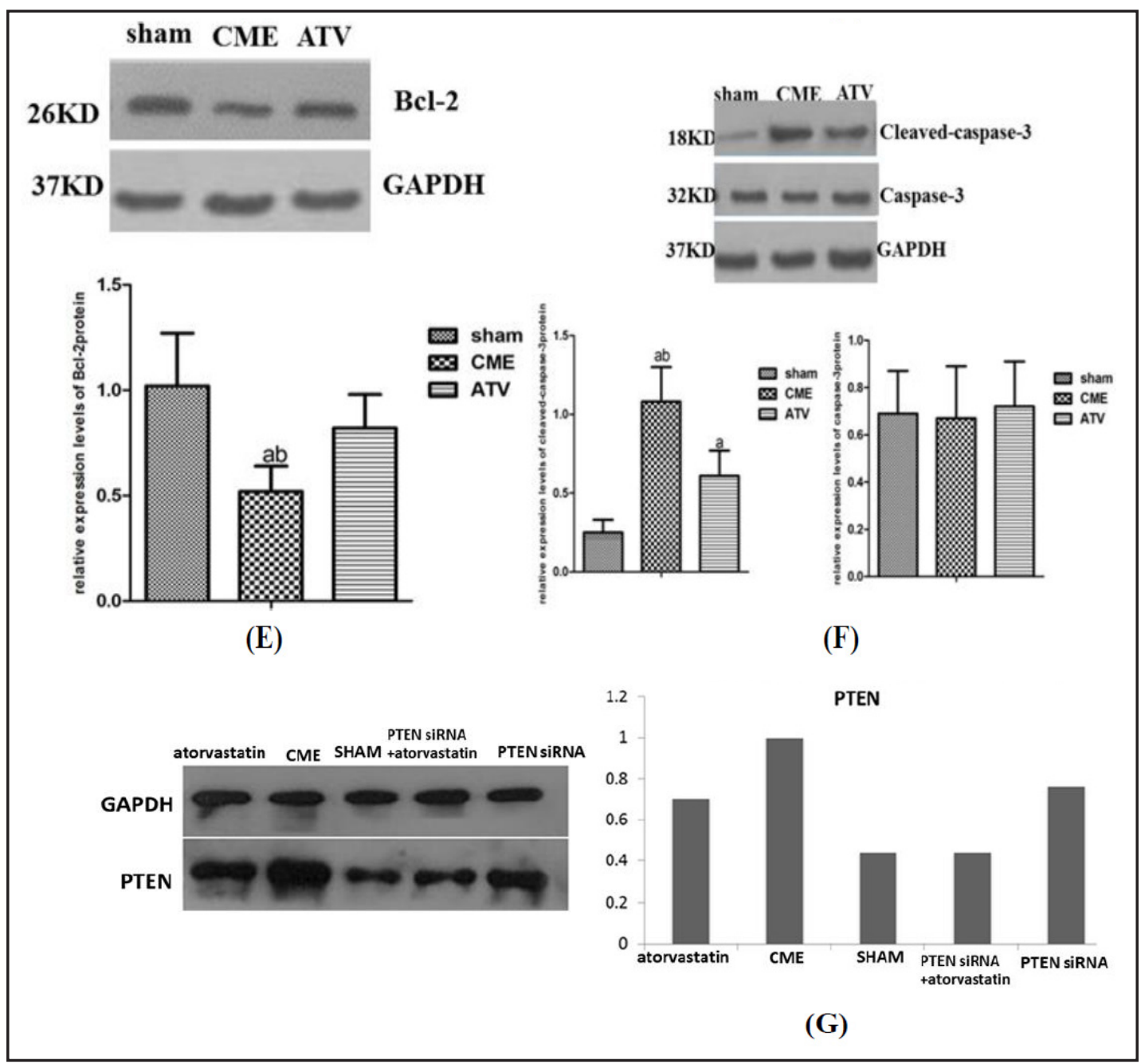

Fig. 7. The molecular cascade after coronary microembolization (CME). CME induced apoptosis via up-regulation of PTEN with PI3K/Akt and cooperating with other Akt-downstream apoptotic signals, such as Bax/Bcl-2 and caspase-3. After using PTEN siRNA or atorvastatin, PI3k/Akt and other downstream genes were affected, followed by the inhibition of the apoptotic cell death process. Sham: sham group; CME: CME: CME group; PTEN siRNA: CME+ PTEN siRNA group; Control siRNA: CME+ Control siRNA group.

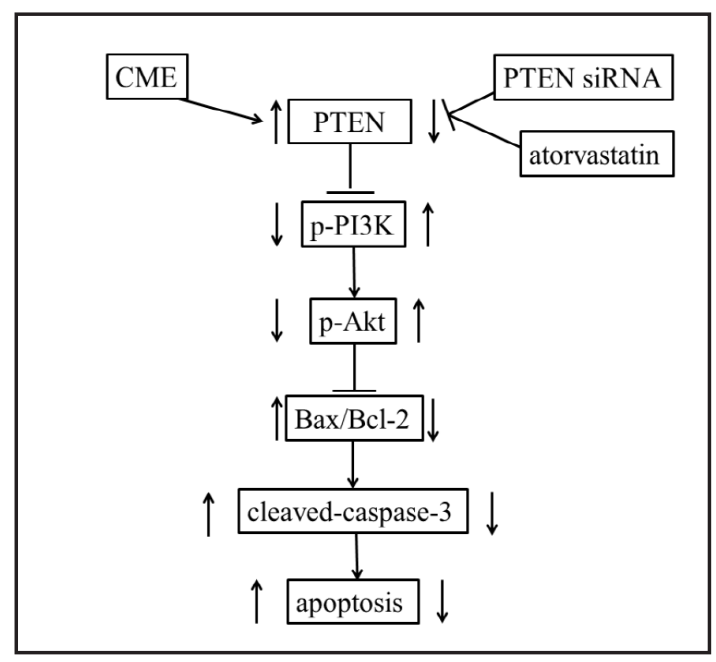

\section{Discussion}

Several novel findings of present study are summarized as followings. First, we showed that PTEN/PI3K/Akt signaling pathway is involved in the pathogenesis of CME-induced myocardial apoptosis. Second, we demonstrated that atorvastatin pretreatment was associated with attenuated myocardial apoptosis and therefore partially reversed the CME 
induced myocardial dysfunction. Third, the potential mechanisms underlying the protective effects of atorvastatin against CME induced cardiac dysfunction may involve the regulation of PTEN/PI3K/Akt signaling pathway.

Results of study highlighted the important role of PTEN/PI3K/Akt in the pathogenesis of CME induced myocardial apoptosis. And, for the first time, our study suggested that atorvastatin may attenuate CME induced cardiac dysfunction by modulation of PTEN/PI3K/ Akt pathway and proposed pretreatment of atorvastatin as a potential therapy for CME related cardiac dysfunction.

PTEN is the most common mutation of tumor suppressor gene. It has been recognized that the most important role of PTEN was to regulate normal developmental processes, including cell growth, adhesion, migration, invasion and apoptosis [15]. Recently, it has been showed that PTEN could be detected in vascular endothelial cells, vascular smooth muscle cells, cardiomyocytes and fibroblasts, and it also served as the key regulator of the pathogenesis of a variety of cardiovascular diseases, such as cardiac hypertrophy, myocardial contractile dysfunction, and cardiomyocytes apoptosis, probably by regulating downstream PI3K/Akt pathways $[12,16]$. A variety of cytokines, growth factors and physical stimulation could activate PI3K so as to phosphorylate and activate Akt, which subsequently functions as a key factor in PI3K/Akt cell signaling pathways and downstream effecter of PI3K [17, 18]. Activated Akt may exert its anti-apoptotic effect via regulation of caspase-3, Bcl-2antagonist of cell death (Bad), forkhead transcription factor, as well as IкB kinase (IKK) [19]. Although early investigations suggested that activation of PI3K/Akt signaling pathway could effectively inhibit cardiomyocyte apoptosis and reduce the extent of myocardial injury [20, 21]. PTEN was found to be highly expressed in myocardial tissues subjected to ischemiareperfusion injury, which inhibits the activation of Akt as a result of myocardial apoptosis. However, the silencing of PTEN expression increases Akt activation, reduces cardiomyocyte apoptosis, and improves cardiac function [22, 23]. However, a previous study about ischemic postconditioning reduces infarct size following ischemia/reperfusion by Skyschally et al. [24] showed that phosphorylation of RISK (reperfusion injury salvage kinases) (Akt) is not causal for protection by ischemic postconditioning. Another studies demonstrated that PI3K/Akt signaling pathway plays an important endogenous cardioprotective role in the pathogenesis of ischemia-reperfusion injury [25, 26]. Skyschally et al. [24] mentioned that a caveat related to almost all studies on the signal transduction of ischemic postconditioning, to date, is the fact that the specific cellular (cardiomyocyte, endothelium, fibroblast, leukocyte) and subcellular (cytosol, mitochondria, nucleus, signalosome) compartment of the respective kinase activation is not known. But, the pathogenesis of CME is distinct from ischemia/reperfusion. And, the results of our study suggested that PTEN/PI3K/Akt pathway was activated in CME and involved in the pathogenesis of myocardial apoptosis.

Atorvastatin had been proposed to be with multiple cardiovascular benefits besides its cholesterol lowering effect, including anti-inflammatory and anti-apoptotic. In recent years, many studies further validated its anti-apoptotic effect in animal models of myocardial ischemia and reperfusion injury [27], coronary atherosclerosis [28], myocardial infarction [29], and renal ischemia-reperfusion injury [30]. More and more studies show that atorvastatin may regulate PTEN/PI3K/Akt cell signaling pathways, although statins may have different regulatory effects in different pathological conditions. Miraglia et al. proved that statins could promote the expression of PTEN protein in tumor cells, inhibit activation of the PI3K/Akt pathway, thereby exert anti -tumor effects [31]. However, Wu et al. found that atorvastatin could inhibit the growth of cardiac myxoma cells, primarily by promoting the expression of PTEN in myxoma cells [32]. Indeed, PI3K/Akt signaling pathway plays an important endogenous cardioprotective role in the pathogenesis of ischemia-reperfusion injury $[25,26]$, and atorvastatin could inhibit the expression of PTEN and enhance activation of PI3K/Akt signaling pathway [33]. In view of the above findings, we proposed that pretreatment of atorvastatin in CME animals may protect against CME related myocardial dysfunction by attenuate myocardial apoptosis via regulation of PTEN/ PI3K/Akt pathways. In this study, we found that atorvastatin pretreatment could reduce 


\section{Cellular Physiology Cell Physiol Biochem 2016;38:207-219

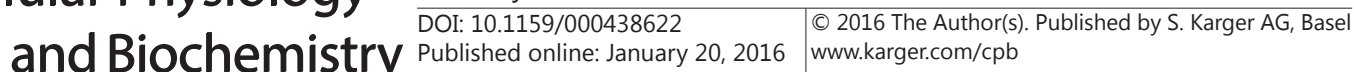 \\ Wang et al.: Atorvastatin is Involved in CME-Induced Myocardial Apoptosis}

CME-induced myocardial injury through inhibiting myocardial apoptosis. Moreover, our study suggested that regulation of PTEN/PI3K/Akt pathway seemed to be an important mechanism underlying the cardioprotective effects of atorvastatin.

In conclusion, the potential therapeutic role of atorvastatin seemed to be related to its regulatory effects on PTEN/PI3K/Akt pathway. However, it should be aware that our researches were performed in swine models and the findings may not be extrapolated directly to humans. Therefore, further researches, especially the translational research in humans are needed to evaluate whether the potential regulatory effects of atorvastatin on PTEN pathways could become a promising treatment strategy for CME related cardiac dysfunction in clinical scenarios.

\section{Acknowledgements}

This study was supported by a grant from National Natural Science Foundation of China (Grant No. 81260042).

\section{Disclosure Statement}

The authors declare no conflicts of interest.

\section{References}

1 Heusch G, Kleinbongard P, Bose D, Levkau B, Haude M, Schulz R, Erbel R: Coronary microembolization: From bedside to bench and back to bedside. Circulation 2009;120:1822-1836.

2 Dorge H, Schulz R, Belosjorow S, Post H, van de Sand A, Konietzka I, Frede S, Hartung T, Vinten-Johansen J, Youker KA, Entman ML, Erbel R, Heusch G: Coronary microembolization: The role of tnf-alpha in contractile dysfunction. J Mol Cell Cardiol 2002;34:51-62.

3 Zhang QY, Ge JB, Chen JZ, Zhu JH, Zhang LH, Lau CP, Tse HF: Mast cell contributes to cardiomyocyte apoptosis after coronary microembolization. J Histochem Cytochem 2006;54:515-523.

4 Dorge H, Neumann T, Behrends M, Skyschally A, Schulz R, Kasper C, Erbel R, Heusch G: Perfusioncontraction mismatch with coronary microvascular obstruction: Role of inflammation. Am J Physiol Heart Circ Physiol 2000;279:H2587-2592.

5 Thielmann M, Dorge H, Martin C, Belosjorow S, Schwanke U, van De Sand A, Konietzka I, Buchert A, Kruger A, Schulz R, Heusch G: Myocardial dysfunction with coronary microembolization: Signal transduction through a sequence of nitric oxide, tumor necrosis factor-alpha, and sphingosine. Circ Res 2002;90:807813.

6 Wang J, Li L, Su Q Zhou Y, Chen H, Ma G, Liu T, Tang Z, Liu Y: The involvement of phosphatase and tensin homolog deleted on chromosome ten (pten) in the regulation of inflammation following coronary microembolization. Cell Physiol Biochem 2014;33:1963-1974.

7 Su Q Li L, Zhou Y, Wang J, Liu Y, Ma G: Induction of myocardial pdcd4 in coronary microembolizationrelated cardiac dysfunction: Evidence from a large-animal study. Cell Physiol Biochem 2014;34:533-542.

8 Qiu R, Cai A, Dong Y, Zhou Y, Yu D, Huang Y, Zheng D, Rao S, Feng Y, Mai W: Sdf-1alpha upregulation by atorvastatin in rats with acute myocardial infarction via nitric oxide production confers anti-inflammatory and anti-apoptotic effects. J Biomed Sci 2012;19:99.

9 Herrmann J, Lerman A, Baumgart D, Volbracht L, Schulz R, von Birgelen C, Haude M, Heusch G, Erbel R: Preprocedural statin medication reduces the extent of periprocedural non-q-wave myocardial infarction. Circulation 2002;106:2180-2183.

10 Li L, Su Q, Wang Y, Dai R, Lu Y, Su B, Zhao Y: Effect of atorvastatin (lipitor) on myocardial apoptosis and caspase-8 activation following coronary microembolization. Cell Biochem Biophys 2011;61:399-406.

11 Latronico MV, Costinean S, Lavitrano ML, Peschle C, Condorelli G: Regulation of cell size and contractile function by akt in cardiomyocytes. Ann N Y Acad Sci 2004;1015:250-260.

12 Oudit GY, Penninger JM: Cardiac regulation by phosphoinositide 3-kinases and pten. Cardiovasc Res 2009;82:250-260. 


\section{Cellular Physiology Cell Physiol Biochem 2016;38:207-219

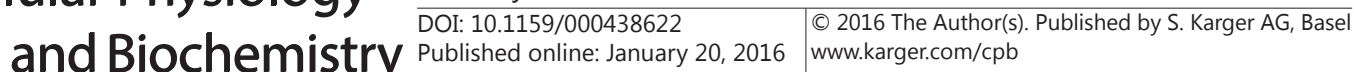 \\ Wang et al.: Atorvastatin is Involved in CME-Induced Myocardial Apoptosis}

13 Breuckmann F, Nassenstein K, Bucher C, Konietzka I, Kaiser G, Konorza T, Naber C, Skyschally A, Gres P, Heusch G, Erbel R, Barkhausen J: Systematic analysis of functional and structural changes after coronary microembolization: A cardiac magnetic resonance imaging study. JACC Cardiovasc Imaging 2009;2:121130.

14 Carlsson M, Wilson M, Martin AJ, Saeed M: Myocardial microinfarction after coronary microembolization in swine: Mr imaging characterization. Radiology 2009;250:703-713.

15 Yamada KM, Araki M: Tumor suppressor pten: Modulator of cell signaling, growth, migration and apoptosis. J Cell Sci 2001;114:2375-2382.

16 Oudit GY, Sun H, Kerfant BG, Crackower MA, Penninger JM, Backx PH: The role of phosphoinositide-3 kinase and pten in cardiovascular physiology and disease. J Mol Cell Cardiol 2004;37:449-471.

17 De Luca A, Maiello MR, D'Alessio A, Pergameno M, Normanno N: The ras/raf/mek/erk and the pi3k/akt signalling pathways: Role in cancer pathogenesis and implications for therapeutic approaches. Expert Opin Ther Targets 2012;16:S17-27.

18 Aksamitiene E, Kiyatkin A, Kholodenko BN: Cross-talk between mitogenic ras/mapk and survival pi3k/akt pathways: A fine balance. Biochem Soc Trans 2012;40:139-146.

19 Torella D, Rota M, Nurzynska D, Musso E, Monsen A, Shiraishi I, Zias E, Walsh K, Rosenzweig A, Sussman MA, Urbanek K, Nadal-Ginard B, Kajstura J, Anversa P, Leri A: Cardiac stem cell and myocyte aging, heart failure, and insulin-like growth factor-1 overexpression. Circ Res 2004;94:514-524.

20 Wang Z, Zhang H, Xu X, Shi H, Yu X, Wang X, Yan Y, Fu X, Hu H, Li X, Xiao J: Bfgf inhibits er stress induced by ischemic oxidative injury via activation of the pi3k/akt and erk1/2 pathways. Toxicol Lett 2012;212:137146.

21 Ye Z, Guo Q, Xia P, Wang N, Wang E, Yuan Y: Sevoflurane postconditioning involves an up-regulation of hif-1alpha and ho-1 expression via pi3k/akt pathway in a rat model of focal cerebral ischemia. Brain Res 2012;1463:63-74.

22 Muslin AJ: Akt2: A critical regulator of cardiomyocyte survival and metabolism. Pediatr Cardiol 2011;32:317-322.

23 Crackower MA, Oudit GY, Kozieradzki I, Sarao R, Sun H, Sasaki T, Hirsch E, Suzuki A, Shioi T, Irie-Sasaki J, Sah R, Cheng HY, Rybin VO, Lembo G, Fratta L, Oliveira-dos-Santos AJ, Benovic JL, Kahn CR, Izumo S, Steinberg SF, Wymann MP, Backx PH, Penninger JM: Regulation of myocardial contractility and cell size by distinct pi3k-pten signaling pathways. Cell 2002;110:737-749.

24 Skyschally A, van Caster P, Boengler K, Gres P, Musiolik J, Schilawa D, Schulz R, Heusch G: Ischemic postconditioning in pigs: No causal role for risk activation. Circ Res 2009;104:15-18.

25 Tong H, Chen W, Steenbergen C, Murphy E: Ischemic preconditioning activates phosphatidylinositol-3kinase upstream of protein kinase c. Circ Res 2000;87:309-315.

26 Mocanu MM, Bell RM, Yellon DM: Pi3 kinase and not p42/p44 appears to be implicated in the protection conferred by ischemic preconditioning. J Mol Cell Cardiol 2002;34:661-668.

27 Bi XY, He X, Zhao M, Yu XJ, Zang WJ: Role of endothelial nitric oxide synthase and vagal activity in the endothelial protection of atorvastatin in ischemia/reperfusion injury. J Cardiovasc Pharmacol 2013;61:391-400.

28 Li Y, Li J, Cui L, Lai Y, Yao Y, Zhang Y, Pang X, Wang J, Liu X: Inhibitory effect of atorvastatin on age-induced hcaec apoptosis by upregulating hsf-1 protein. Int J Biol Macromol 2013;57:259-264.

29 Tang XL, Sanganalmath SK, Sato H, Bi Q Hunt G, Vincent RJ, Peng Y, Shirk G, Dawn B, Bolli R: Atorvastatin therapy during the peri-infarct period attenuates left ventricular dysfunction and remodeling after myocardial infarction. PLoS One 2011;6:e25320.

30 Haylor JL, Harris KP, Nicholson ML, Waller HL, Huang Q Yang B: Atorvastatin improving renal ischemia reperfusion injury via direct inhibition of active caspase-3 in rats. Exp Biol Med (Maywood) 2011;236:755763.

31 Miraglia E, Hogberg J, Stenius U: Statins exhibit anticancer effects through modifications of the pakt signaling pathway. Int J Oncol 2012;40:867-875.

32 Wu XL, Yang DY, Tan DJ, Yao HC, Chai W, Peng L: Inhibitory effect of atorvastatin on the cell growth of cardiac myxomas via the pten and phlpp2 phosphatase signaling pathway. Oncol Rep 2013;30:757-762.

33 Mensah K, Mocanu MM, Yellon DM: Failure to protect the myocardium against ischemia/reperfusion injury after chronic atorvastatin treatment is recaptured by acute atorvastatin treatment: A potential role for phosphatase and tensin homolog deleted on chromosome ten? J Am Coll Cardiol 2005;45:1287-1291. 